\title{
A STUDY OF MULTIDRUG-RESISTANT ACINETOBACTER BAUMANNII- A SUSCEPTIBILITY PATTERN AND POSSIBLE RISK FACTORS IN A TERTIARY CARE HOSPITAL OF CHHATTISGARH
}

\author{
Nikita Sherwani ${ }^{1}$, Rekha Barapatre ${ }^{2}$, Arvind Neral ${ }^{3}$, Rachana Singh ${ }^{4}$, Neeta Gade 5
}

${ }_{1}^{1}$ Associate Professor, Department of Microbiology, Pt. JNM Medical College, Raipur, Chhattisgarh, India.

${ }^{2}$ Assistant Professor, Department of Microbiology, Pt. JNM Medical College, Raipur, Chhattisgarh, India.

3Professor, Department of Microbiology, Pt. JNM Medical College, Raipur, Chhattisgarh, India.

${ }^{4}$ Assistant Professor, Department of Microbiology, Pt. JNM Medical College, Raipur, Chhattisgarh, India.

${ }_{5}^{5}$ Assistant Professor, Department of Microbiology, Pt. JNM Medical College, Raipur, Chhattisgarh, India.

\section{ABSTRACT}

\section{BACKGROUND}

The resistance of Acinetobacter varies widely geographically and between various units of the same hospital at various time points. The variations in Acinetobacter resistance demands a periodic surveillance of these pathogens to achieve appropriate selection of therapy. Due to unpredictable multidrug resistance patterns of clinical strains of Acinetobacter baumannii, it is important to know the prevalent susceptibility profiles as well as various risk factors associated with multidrug resistance.

The aim of this study is to determine the proportion of Acinetobacter infections, susceptibility patterns and the possible risk factors for the multidrug resistance.

\section{MATERIALS AND METHODS}

This study was conducted in a tertiary care hospital over a period of 18 months. Acinetobacter species were isolated from a range of clinical samples (ICU, wards, outpatient departments) received in Department of Microbiology. The antibiotic susceptibility was determined by the standard disc diffusion method.

\section{RESULTS}

Out of total 1581 positive samples, 4.7\% were found to be Acinetobacter. The most predominant species was Acinetobacter baumannii complex (72.8\%). Samples showed the maximum resistance to piperacillin (49\%), ceftazidime (48\%), amikacin (46\%), cefepime (41\%), ciprofloxacin (27\%), imipenem and netilmicin (33\%). Antibiotic resistance was higher in the samples from Intensive Care Units (ICUs) of the hospital. Statistical analysis showed the following risk factors for Multidrug Resistance (MDR) A.baumannii infection: the occurrence of ischaemic heart disease patients $(\mathrm{OR}=2.12, \mathrm{p}$-value 0.04$)$, mechanical ventilation $(\mathrm{OR}=$ 2.84, $\mathrm{p}$-value $=0.001)$ and usage of home-based antimicrobial treatment $(\mathrm{OR}=2.47, \mathrm{p}$-value $=0.02)$.

\section{CONCLUSION}

This study clearly documents a high prevalence rate of infections as well as antibiotic resistance. The occurrence of multidrug resistant infections was associated with the risk factors, which can be prevented or treated early.

\section{KEY WORDS}

Acinetobacter, Multidrug Resistance, ICU, Chhattisgarh.

HOW TO CITE THIS ARTICLE: Sherwani N, Barapatre R, Neral A, et al. A study of multidrug-resistant acinetobacter baumannii- a susceptibility pattern and possible risk factors in a tertiary care hospital of Chhattisgarh. J. Evolution Med. Dent. Sci. 2018;7(36): 3935-3939, DOI: 10.14260/jemds/2018/880

\section{BACKGROUND}

Acinetobacter baumannii is an aerobic, non-motile gramnegative bacillus and an important cause of hospital acquired infections.[1] Acinetobacter pose a great problem to medical fraternity, mainly because of its substantial resistance pattern and also to the range of infections caused by it (local wound infection, pneumonia, bacteraemia, meningitis).[2] The transmission of these infections is credited to the versatility of Acinetobacter growing at various temperatures and $\mathrm{pH}$ conditions using both carbon and energy sources as well as its propensity to survive in hospital environment. [3]

'Financial or Other Competing Interest': None.

Submission 25-07-2018, Peer Review 18-08-2018,

Acceptance 25-08-2018, Published 03-09-2018.

Corresponding Author:

Dr. Rekha Barapatre,

Department of Microbiology,

Pt. JNM Medical College, Jail Road,

Raipur-492001, Chattisgarh, India.

E-mail: gonnaderekha@gmail.com

DOI: $10.14260 /$ jemds $/ 2018 / 880$

\section{(c) $(1)$}

Acinetobacter baumannii is the most clinically important bacteria belonging to the genus of Acinetobacter. ${ }^{[4]}$ The nosocomial infections acquired by Acinetobacter baumannii are associated with increased morbidity and mortality, especially in critically ill patients.[5] Carbapenems used to be effective against multidrug-resistant $\mathrm{A}$. baumannii, but it has become more and more difficult to treat this pathogen due to its pan resistance. According to Centres for Disease Control and Prevention (CDC), the resistance of A. baumannii against carbapenems has increased from $9 \%$ in 1995 to $40 \%$ in 2004.[6]

The ability of A. baumannii to show resistance to common disinfectants and its survival capacity on dry surfaces makes it a troublesome organism to be eradicated from the hospitals. Latest data shows that the Multidrug Resistance (MDR) of A. baumannii varies from 48 to $85 \%$ of clinical sample. Asia, Eastern Europe and Latin America have the greatest burden of this resistance problem. Its resistance against almost all antimicrobials is increasing and pose a great problem to the clinicians with increasing infections and limited options to control its spread. To fight this rising trend 
of multidrug resistance pattern, there is a need to develop new antimicrobial approaches along with appropriate planning for cost effective health care policies in the developing countries like India.[7,8] Hence, there is a need for the research on the proportions and trends in antibiotic resistance among Acinetobacter species. More studies are required to gain more knowledge about the development and spread of antibiotic resistance in India. In this study, we have examined the proportions of and trends in MultidrugResistance (MDR) among Acinetobacter spp. isolates from a tertiary care hospital of India during the period 2016 - 2018.

\section{MATERIALS AND METHODS}

It was a prospective observational study performed in Department of Microbiology, Pt. Jawaharlal Memorial Medical College and associated Bhimrao Ambedkar Memorial Hospital, Raipur, Chattisgarh. It is a tertiary care, 1500 bedded teaching hospital. Sample included a range of clinical specimens (blood, CSF, pus, other body fluids) which were sent to the laboratory by all the departments including ICU, Medicine, Surgery, Obstetrics and Gynaecology, Paediatrics and various Outpatient Departments (OPDs). This study was conducted over a period of eighteen months from August 2016 to January 2018. As part of this research work, over a period of one and a half year around 3100 clinical samples were studied to isolate various Acinetobacter species by a simplified phenotypic identification method and also the antibiotic susceptibility pattern of these isolates was determined as per Clinical and Laboratory Standards Institute guidelines including following antibiotics: Ceftazidime, cefepime, piperacillin, ciprofloxacin, imipenem and amikacin. ${ }^{[9]}$ Kirby-Bauer disk diffusion method was used for antimicrobial susceptibility testing.[10] Acinetobacter are non-motile bacilli or coccobacilli. Identification of the genus was done on the basis of its morphology, gram staining, nonmotility, positive catalase test and negative oxidase test.[11]

\section{Statistical Methods}

Data were analysed using SPSS version 21. Percentage and proportions were calculated for the obtained data. Univariate analyses was done using Chi-square test to see the difference in antibiotic sensitivity pattern of Acinetobacter species isolated from ICU and non-ICU samples. Logistic regression was done to identify the demographic and individual variables associated with the occurrence of MDR Acinetobacter baumannii infection. The independent variables which were explored in regression analyses were: age $(<50,>50)$, gender (female, male); smoking (yes, no); ischaemic heart disease (yes, no); lung disease (yes, no); mechanical ventilation (yes, no); antimicrobial treatment at home (yes, no) and ICU stay ( $<30$ days, $>30$ days). The association between the MDR Acinetobacter baumannii infection and selected characteristics, which have been described above was analysed by means of logistic regression and expressed as Adjusted Odds Ratio (AOR) with a confidence interval at $95 \%(95 \%, \mathrm{CI})$. The AOR measures the strength of association between the characteristics of the study population and occurrence of MDR Acinetobacter baumannii infection. Exploratory data analyses were conducted to examine the relationship among dependent variable (occurrence of Acinetobacter infection) and other predictive variables using appropriate regression with backward elimination approach. Dummy coding of categorical variables $(1,0)$ was done to fit them in predictive modelling. Also, before finalising the fitted model, the correlation coefficients of all the variables were investigated to ensure the significance of each predictive variable. It is important to state that to finalise the model all the requisite assumptions, i.e. linearity, normality etc. were met, for all analyses p-value of $<0.05$ was considered to be statistically significant.

\section{RESULTS}

Over a period of one and a half year, out of the total 3100 samples 1581 (51\%) were culture positive. Gram negative cultures were found to be around $44 \%$ of the total positive cultures with Acinetobacter forming $4.7 \%$ of the total positive cultures. Table 1 shows the various species of Acinetobacter identified from clinical samples. Out of the total Acinetobacter cultures, Acinetobacter baumannii complex represented the highest number $(72.8 \%)$ of isolates followed by Acinetobacter lwoffii (13.1\%), A. haemolyticus (7.7\%), A. junii $(4.2 \%)$ and $A$. radioresistens $(2.2 \%)$. The maximum number (44\%) of Acinetobacter species were isolated from Intensive care units followed by surgical wards (19\%), medicine ward (15\%), orthopaedics (11\%), burn unit (4\%), paediatrics $(3 \%)$, gynaecology ward $(2 \%)$ and outpatient departments (2\%).

\begin{tabular}{|c|c|}
\hline Acinetobacter Species & Isolates (\%) \\
\hline Acinetobacter baumannii & 72.8 \\
\hline Acinetobacter lwoffii & 13.1 \\
\hline Acinetobacter haemolyticus & 7.7 \\
\hline Acinetobacter junii & 4.2 \\
\hline Acinetobacter radioresistens & 2.2 \\
\hline Table 1. Acinetobacter species identified from Clinical \\
Samples \\
\hline
\end{tabular}

Table 2 shows the antimicrobial sensitivity patterns of Acinetobacter along with comparison between ICU and other samples (other wards and outpatient departments). In the present study, Acinetobacter strains were found to be more resistant to piperacillin (49\%), ceftazidime (48\%), amikacin (46\%), cefepime (41\%), ciprofloxacin (27\%), imipenem and netilmicin (33\%). ICU samples were found to have comparatively higher resistance against all antibiotics as compared to the non-ICU samples and the difference was statistically significant ( $p$-value $<0.05$ ).

\begin{tabular}{|c|c|c|c|c|}
\hline Antibiotic & $\begin{array}{c}\text { Sensitivity } \\
\text { Patterns } \\
\text { (\%) }\end{array}$ & $\begin{array}{c}\text { Susceptibility } \\
\text { in ICU } \\
\text { Samples (\%) }\end{array}$ & $\begin{array}{c}\text { Susceptibility } \\
\text { in non-ICU } \\
\text { Samples (\%) }\end{array}$ & $\begin{array}{c}\text { P } \\
\text { value }\end{array}$ \\
\hline Imipenem & 77 & 65 & 88 & 0.001 \\
\hline Ciprofloxacin & 73 & 62 & 84 & 0.001 \\
\hline Ceftazidime & 52 & 39 & 65 & 0.002 \\
\hline Cefepime & 59 & 38 & 79 & $<0.001$ \\
\hline Piperacillin & 51 & 32 & 70 & $<0.001$ \\
\hline Netilmicin & 77 & 66 & 87 & 0.004 \\
\hline Amikacin & 56 & 38 & 74 & $<0.001$ \\
\hline
\end{tabular}

Table 2. Univariate Analysis of Antimicrobial sensitivity patterns of Acinetobacter species in ICU and non-ICU Samples

Table 3 shows the Logistic regression analysis of patient's characteristics and possible risk factors for MultidrugResistant (MDR) Acinetobacter baumannii infection, 
according to patients' various characteristics and relevant history. Although, MDR Acinetobacter baumannii infection was found to be more common in the patients above fifty years of age; however, the difference was not significant. Similarly, male gender, smoking history and concomitant lung disease were also found to be associated with the infection. The MDR Acinetobacter baumannii infection was significantly associated with the occurrence of ischaemic heart disease patients $(\mathrm{OR}=2.12$, $\mathrm{p}$-value $=0.04)$. Other significant risk factors were found to be mechanical ventilation $(\mathrm{OR}=2.84$, $\mathrm{p}$ value $=0.001$ ) and usage of home-based antimicrobial treatment $(\mathrm{OR}=2.47, \mathrm{p}$-value $=0.02)$. Acinetobacter baumannii strains were found to have shown more resistance in ICU samples, but the length of the ICU stay was not significantly associated with the infection.

\begin{tabular}{|c|c|c|c|}
\hline Characteristics & $\begin{array}{l}\text { Odd's } \\
\text { Ratio } \\
\end{array}$ & CI & P-value \\
\hline $\begin{aligned} & \text { Age } \\
&-<50 \text { years } \\
&->50 \text { years } \\
&\end{aligned}$ & 1.13 & $0.85-1.31$ & 0.15 \\
\hline $\begin{array}{cl}\text { Gender } & \\
- & \text { Female } \\
- & \text { Male } \\
\end{array}$ & 1.36 & $0.91-2.31$ & 0.26 \\
\hline \begin{tabular}{|cc} 
Smoking & \\
- & Yes \\
- & No \\
\end{tabular} & 0.92 & $0.86-2.92$ & 0.18 \\
\hline \begin{tabular}{|cc|}
\multicolumn{2}{|c|}{ Ischaemic heart disease } \\
$-\quad$ Yes \\
$-\quad$ No \\
\end{tabular} & 2.12 & $.31-5.82$ & 0.04 \\
\hline \begin{tabular}{rr}
\multicolumn{2}{|l}{ Lung Disease } \\
$-\quad$ Yes \\
$-\quad$ No \\
\end{tabular} & 1.61 & $0.72-2.84$ & 0.17 \\
\hline \begin{tabular}{|c|} 
Mechanical ventilation \\
$-\quad$ Yes \\
$-\quad$ No \\
\end{tabular} & 2.84 & $1.6-6.24$ & 0.001 \\
\hline $\begin{array}{l}\text { Home antimicrobial } \\
\text { treatment } \\
-\quad \text { Yes } \\
-\quad \text { No } \\
\end{array}$ & 2.47 & $1.43-6.12$ & 0.02 \\
\hline \begin{tabular}{|cc} 
ICU stay & \\
- & $<30$ days \\
- & $>30$ days
\end{tabular} & 0.98 & $0.84-1.56$ & 0.31 \\
\hline $\begin{array}{r}\text { Table 3. Logistic } \\
\text { characteristics and po } \\
\text { bau }\end{array}$ & niis & $\begin{array}{l}\text { alysis of pa } \\
\text { tors for Acil } \\
\text { ion }\end{array}$ & $\begin{array}{l}\text { ents } \\
\text { tobacter }\end{array}$ \\
\hline
\end{tabular}

\section{DISCUSSION}

During the last few decades, there is a substantial increase in incidence of microbial infections and due to perpetual use of antimicrobial drugs in treating these infections, emergence of antimicrobial resistance among these microorganisms is common. Multidrug resistance is a well-documented phenomenon in clinical strains of Acinetobacter[12] and emerging as one of the robust healthcare-associated infections to control and treat as well. Multidrug-resistant Acinetobacter has been found to be the cause of various outbreaks in ICUs, neonatal ICUs and burn units.[13,14,15] It is responsible for spreading the infection among susceptible patients with central intravenous catheters and respiratory devices and those who are admitted in ICUs.[13]

In our study, we tried to identify all the isolates of Acinetobacter upto species level. Acinetobacter baumannii complex was the most predominant (72.8\%) species followed by A. lwoffii (13.1\%), A. haemolyticus (7.7\%), A. junii (4.2\%) and A. radioresistens (2.2\%). This finding is similar to the other studies, in which A. baumannii complex was the most common of all the species identified in the samples.[16,17]

In our study Acinetobacter strains were found to be resistant to piperacillin (49\%), ceftazidime (48\%), amikacin (46\%), cefepime (41\%), ciprofloxacin (27\%), imipenem and netilmicin (33\%).

Khan MA et al reported that A. baumannii isolates showed a higher degree of resistance to piperacillin (93.1\%), aztreonam $(80.5 \%)$, ticarcillin, ampicillin and tetracycline (76.4\%, each) and cefotaxime (75\%), except amikacin which showed low rate of resistance (40.3\%). [18]

The ICU samples showed more resistance to all the antibiotics as compared to non-ICU samples. These results are also comparable to that reported by Gupta $\mathrm{N}$ et al, in which the susceptibility of ICU samples to almost all the antibiotics was significantly lower than non-ICU samples.[17]

The individual risk factors for the MDR A. baumannii infection were found to be the presence of ischaemic heart disease, mechanical ventilation and home antimicrobial treatment. This association of risk factors to MDR A. baumannii infection has been demonstrated by Abbo A et al, who hypothesised this association to be attributed to the severity of patient's condition, invasive device usage, altered normal flora which can lead to the emergence of multidrug resistance.[19]

In our study, most of the Acinetobacter isolates were obtained from respiratory samples and also mechanical ventilation was found to be a significant risk factor for multidrug resistant samples. This finding is comparable with the results of other studies, in which respiratory samples were responsible for most of the Acinetobacter isolates.[20,21,22,23] The explanation for the higher prevalence of multidrug resistant isolates from respiratory samples could be that the Acinetobacter strains, which cause ventilator-associated pneumonia or other healthcareassociated pneumoniae are more frequently exposed to antibiotics compared to strains causing other types of infection. Although, Odsbu I et al found in their study that significantly higher proportions of multidrug resistant isolates were from respiratory samples, the proportions of multidrug resistant isolates from other specimen types were still high, which indicates that empirical treatment of all types of infections caused by Acinetobacter is difficult.[24] Acinetobacter spp. have a natural multidrug resistant phenotype.[25] High proportions of multidrug resistant isolates were found in our study, comparable are the findings reported in various studies worldwide.[26,27,28] There was also high proportion of carbapenem resistant isolates observed in our hospital over a period of eighteen months. This finding is also consistent with that of the study done in a tertiary care centre of western India in similar settings, which reported $42 \%$ carbapenem-resistant Acinetobacter spp. isolates from patients admitted to the intensive care unit.[29]

\section{CONCLUSION}

Acinetobacter species is one of the emerging and global nosocomial pathogens with a high occurrence in hospitals. Our study also identified certain risk factors, which were found to be significantly associated and need to be taken care of to prevent multidrug resistance. Wise and effective use of antibiotics, effective surveillance of resistance and adherence to infection control practices, perhaps are the key factors that 
may prevent the development and spread of multidrug resistance among the prevalent strains of acinetobacter species.

\section{Strengths and Limitations}

This study was a prospective study conducted over a period of eighteen months. We collected the longitudinal surveillance data and analysed the samples in the same laboratory, which add to the few research studies done in India. Analysing the samples in same laboratory reduces the probability of any bias due to methodological causes. The limitation of the study was that we tested the isolates for few antimicrobials, which can lead to either overestimation or underestimation of the number of multidrug resistant samples.

\section{REFERENCES}

[1] Manikal VM, Landman D, Saurina G, et al. Endemic carbapenem-resistant Acinetobacter species in Brooklyn, New York: citywide prevalence, interinstitutional spread and relation to antibiotic usage. Clin Infect Dis 2000;31(1):101-6.

[2] Rit K, Saha R. Multidrug-resistant acinetobacter infection and their susceptibility patterns in a tertiary care hospital. Nigerian Medical Journal 2012;53(3):126-8.

[3] Vashist J, Rajeswari MR. Acinetobacter baumannii: emerging nosocomial pathogen. ENVIS CENTRE Newsletter

2011;9(1) http://www.envismadrasuniv.org/nl20111Acinetoba cterbaumanniiemergingnosocomialpathogen.html

[4] Peleg AY, Seifert H, Paterson DL. Acinetobacter baumannii: emergence of a successful pathogen. Clinical Microbiology Reviews 2008;21(3):538-82.

[5] Abbott Iain, Cerqueira GM, Bhuiyan $\mathrm{S}$, et al. Carbapenem resistance in Acinetobacter. Laboratory challenges, mechanistic insights and therapeutic strategies. Expert Rev Anti Infect Ther 2013;11(4):395-409.

[6] Munoz-Price LS, Weinstein RA. Acinetobacter infection. New England Journal of Medicine 2008;358(12):1271-81.

[7] Hua X, Zhou H, Jiang Y, et al. Genome sequences of two multidrug-resistant Acinetobacter baumannii strains isolated from a patient before and after treatment with tigecycline. J Bacteriol 2012;194(24):6979-80.

[8] Wan Nor Amilah WA, Izani NNJ, Ng WK, et al. A simple screening test for the detection of metallo-Betalactamase-producing pseudomonas aeruginosa and acinetobacter in a tertiary care hospital. Trop Biomed 2012;29(4):588-97.

[9] Clinical and Laboratory Standards Institute. Performance Standards for Antimicrobial Susceptibility Testing: Seventeenth Informational Supplement. CLSI document M100-S17. Wayne, PA, USA: CLSI, 2007.

[10] Winn WC, Allen SD, Janda WM, et al. Taxonomy, biochemical characteristics and clinical significance of medically important non-fermenters. In: Darcy P, Peterson N, eds. Koneman's Colour Atlas and Textbook of Diagnostic Microbiology. 6 $6^{\text {th }}$ edn. Philadelphia: Lippincott Williams and Wilkins 2006: p. 353-5.
[11] Lone R, Shah A, Kadri SM, et al. Nosocomial multi-drug resistant acinetobacter infections-clinical findings, risk factors and demographic characteristics. Bangladesh J Med Microbiol 2009;03(1):34-8.

[12] Pardesi KR, Yavankar SP, Chopade BA. Plasmid distribution \& antimicrobial susceptibility patterns of acinetobacter genospecies from healthy skin of a tribal population in western India. Indian J Med Res 2007;125(1):79-88.

[13] Xia Y, Lu C, Zhao J, et al. A bronchofiberoscopyassociated outbreak of multidrug-resistant acinetobacter baumannii in an intensive care unit in Beijing, China. BMC Infect Dis 2012;12:335.

[14] Saleem AF, Ahmed I, Mir F, et al. Pan-resistant acinetobacter infection in neonates in Karachi, Pakistan. J Infect Dev Ctries 2009;4(1):30-7.

[15] Merino M, Acosta J, Poza $M$, et al. OXA-24 carbapenemase gene flanked by XerC/XerD-like recombination sites in different plasmids from different acinetobacter species isolated during a nosocomial outbreak. Antimicrob Agents Chemother 2010;54(6):2724-7.

[16] Fayyaz M, Khan IU, Hussain A, et al. Frequency and antimicrobial susceptibility pattern of acinetobacter species isolated from pus and pus swab specimens. Journal of the College of Physicians and Surgeons Pakistan 2015;25(5):346-9.

[17] Gupta N, Gandham N, Jadhav S, et al. Isolation and identification of acinetobacter species with special reference to antibiotic resistance. Journal of Natural Science, Biology and Medicine 2015;6(1):159-62.

[18] Khan MA, Mahomed MF, Ashshi AM, et al. Drug resistance patterns of acinetobacter baumannii in Makkah, Saudi Arabia. Pak J Med Res 2012;51(4):12731.

[19] Abbo A, Navon-Venezia S, Hammer-Muntz O, et al. Multidrug-resistant acinetobacter baumannii. Emerging Infectious Diseases 2005;11(1):22-9.

[20] Sivaranjani V, Umadevi S, Srirangaraj S, et al. Multidrug resistant acinetobacter species from various clinical samples in a tertiary care hospital from South India. Australas Med J 2013;6(12):697-700.

[21] Sohail M, Rashid A, Aslam B, et al. Antimicrobial susceptibility of acinetobacter clinical isolates and emerging antibiogram trends for nosocomial infection management. Rev Soc Bras Med Trop 2016;49(3):3004.

[22] Uwingabiye J, Frikh M, Lemnouer A, et al. Acinetobacter infections prevalence and frequency of the antibiotics resistance: comparative study of intensive care units versus other hospital units. Pan Afr Med J 2016;23:191.

[23] Zilberberg MD, Kollef MH, Shorr AF. Secular trends in acinetobacter baumannii resistance in respiratory and blood stream specimens in the United States, 2003 to 2012: a survey study. J Hosp Med 2016;11(1):21-6.

[24] Odsbu I, Khedkar S, Khedkar U, et al. High proportions of multidrug-resistant acinetobacter spp. Isolates in a district in western India: a four-year antibiotic susceptibility study of clinical isolates. International journal of Environmental Research and Public Health 2018;15(1):E153. 
[25] Dijkshoorn L, Nemec A, Seifert H. An increasing threat in hospitals: Multidrug-resistant acinetobacter baumannii. Nat Rev Microbiol 2007;5(12):939-51.

[26] Lob SH, Hoban DJ, Sahm DF, et al. Regional differences and trends in antimicrobial susceptibility of acinetobacter baumannii. Int $\mathrm{J}$ Antimicrob Agents 2016;47(4):317-23.

[27] Chung DR, Song JH, Kim SH, et al. High prevalence of multidrug-resistant non-fermenters in hospitalacquired pneumonia in Asia. Am J Respir Crit Care Med 2011;184(12):1409-17.
[28] Sader HS, Farrell DJ, Flamm RK, et al. Antimicrobial susceptibility of Gram-negative organisms isolated from patients hospitalized in intensive care units in United States and European hospitals (2009-2011). Diagn Microbiol Infect Dis 2014;78(4):443-8.

[29] Khajuria A, Praharaj AK, Kumar M, et al. Molecular characterization of carbapenem resistant isolates of acinetobacter baumannii in an intensive care unit of a tertiary care centre at Central India. J Clin Diagn Res 2014;8(5):DC38-DC40. 